Article

\title{
Genomic Analysis of Staphylococcus aureus Strains Originating from Hungarian Rabbit Farms Reinforce the Clonal Origin of Various Virulence Types
}

\author{
Zoltán Német ${ }^{1, *(1)}$, Ervin Albert ${ }^{1}$, Ádám Dán ${ }^{1}$, Gyula Balka ${ }^{1}$, Áron Szenes ${ }^{1}$, Rita Sipos ${ }^{2}$, \\ Szabolcs Bódizs ${ }^{1}$ and Imre Biksi ${ }^{1}$ \\ 1 Department of Pathology, University of Veterinary Medicine Budapest, István u. 2., \\ H-1078 Budapest, Hungary; albert.ervin@univet.hu (E.A.); dan.adam@univet.hu (Á.D.); \\ balka.gyula@univet.hu (G.B.); szenes.aron@univet.hu (Á.S.); szab.bodizs@gmail.com (S.B.); \\ biksi.imre@univet.hu (I.B.) \\ 2 Biomi Ltd., Szent-Györgyi Albert út 4, H-2100 Gödöllő, Hungary; sipos.rita@biomi.hu \\ * Correspondence: nemet.zoltan@univet.hu; Tel.: +36-30-606-8142
}

Received: 14 May 2020; Accepted: 30 June 2020; Published: 2 July 2020

Simple Summary: Staphylococcosis is a major disease in both human and veterinary medicine. In commercial rabbit production, highly virulent variants cause significant economic losses. This study describes the comparative analysis of the whole genome of 51 strains sequenced by our group, and 12 sequences derived from online repositories. The investigation was based on whole-genome sequencing and whole-genome multilocus sequence typing. The typical highly virulent strains showed great similarity with ST121/t645 isolates from Italy, Spain and the UK. The most prevalent genotype in Hungary was an atypical highly virulent variant; these strains form a novel sequence type (ST5993), and belong to three different spa types (t4770, t711 and t2407). Low virulence (LV) strains grouped into two main clusters, two ST1 LV strains formed a separate cluster from the majority forming a clone-like group, despite the five different multilocus sequence typing (MLST) patterns and seven different spa types. Some strains in this survey showed genetic polymorphism on more than $50 \%$ of the examined loci, however, even within the same MLST ST group, hundreds of loci showed polymorphism, which could facilitate the very fine differentiation of the strains.

\begin{abstract}
Staphylococcosis is one of the most important infectious diseases in rabbit medicine, especially in commercial farming. Previous studies revealed the existence of virulent variants adapted to rabbits. Typical and atypical, highly virulent as well as low virulent variants have been isolated and reported from industrial units in all major rabbit-meat-producing countries. Preceding the research focused on detecting defined nucleotide sequences, the genome of these organisms as a whole was rarely subjected to scientific investigations. The authors sequenced 51 Staphylococcus strains originating from industrial rabbit farms in Hungary. Another 12 draft genomes of rabbit isolates were constructed from read sequences available in digital repositories, and were compared based on whole-genome multilocus sequence typing. The clonal origin of highly virulent variants is confirmed, the strains from Hungary were closely related with the strains isolated in the UK, Italy, and Spain. Atypical highly virulent strains are the most prevalent in Hungary, they form a separate clonal cluster. The low virulent strains were genetically similar, but more heterogeneous than the highly virulent (HV) and aHV strains even by the traditional MLST typing scheme. Other "non-aureus" Staphylococcus species were also identified.
\end{abstract}

Keywords: Staphylococcus aureus; rabbits; whole genome; wgMLST; spa-typing; MLST 


\section{Introduction}

Several clinical forms of Staphylococcus aureus infections could cause devastating diseases in rabbit farming. Production losses due to mortality, premature elimination and slaughterhouse condemnations can result in a significant decrease in profitability. The pathogen can damage the skin and internal organs; usually subacute-chronic, purulent-necrotic lesions develop in infected animals. Reservoir lesions, e.g., mastitis or sore hocks (pododermatitis ulcerosa) in breeding animals are unable to completely heal. The lasting presence of the pathogen often results in the infection of new-born rabbits, and the consequent septicaemia and mortality seriously hinder production [1]. The contamination of farms with virulent variants of Staphylococcus aureus often calls for radical solutions, usually a complete depopulation and disinfection of the unit [2]. Staphylococcus aureus is a zoonotic agent, and a recent study reported that the rabbits and the workers on a rabbit farm can carry identical clonal types of this pathogenic microorganism [3]. Intensively producing commercial farms usually focus on production and buy young females from genetic centres. The fertility of females decreases over time, and 2-2.5-year-old, otherwise healthy females are slaughtered for meat. In order to maintain the peak production levels on the farm, usually $80-140 \%$ of the breeding flock is replaced each year. Some producers are involved in long-term contracts with suppliers, which in turn creates a stable epidemiological chain. In other cases, the farmer is looking for better production results and thus constantly changes the genetic resources. This creates a constant influx of live animals on all farms, along with the possibility of introducing new pathogenic agents to the population.

The detection of such virulent pathogens in rabbits can be accomplished by routine bacterial culturing, followed by a multiplex polymerase chain reaction (PCR) to detect three virulence determinant genes [4]. Previous studies revealed the existence of highly virulent strains (HV), which were detected in Belgium, France, Greece, Italy, Portugal and Spain [5]. Our research group previously reported the presence of such strains on Hungarian rabbit farms [6]. HV strains were identified as Staphylococcus aureus clonal complex (CC) type 121 (or sequence type 121, ST121), similar strains were known for their increased virulence, and were proven to be globally distributed [7]. A study revealed that a single nonsynonymous nucleotide mutation in the coding region of an integral membrane protein (dltB) was associated with the host adaptation of CC121 from humans to rabbits [8]. Holmes et al. (2016) reported whole-genome sequencing (WGS) results of a Staphylococcus aureus strain belonging to ST121, isolated from a companion rabbit in the UK [9].

Previous studies also reported atypical highly virulent strains (aHV), which were considered virulent based on the severity of the outbreak they caused in Belgium in 1994 [10]. Since two virulence genes were undetectable in these strains using multiplex PCR, this genotype was considered atypical [4]. Decades later, this genotype was found to be the most common genotype in Hungarian meat-producing rabbit populations [6].

Low virulence strains (LV) cause sporadic infections and the prevalence of such strains is difficult to estimate [2]. Previous studies found that LV strains have biochemical properties similar to the isolates of human or poultry origin [11].

Other studies on rabbit-related Staphylococcus isolates focused on different sequence regions [3,12-15], which proved very useful, but only a handful of genes were used to identify and classify different variants of the pathogen. Minor differences in the targeted loci can show the same distinctness as would be measured with profoundly diverse strains, and the mutations of distant variants of a species can also lead to the evolution of the same nucleotide sequence [16].

Whole-genome short read shotgun sequencing of small genomes has become an affordable method on the Illumina platform nowadays. The cost of sequencing the $2.8 \mathrm{Mbp}$ genome of Staphylococcus aureus with high coverage is comparable to 8-10 polymerase chain reactions (PCR) and the following Sanger sequencing, which is needed for multilocus sequence typing (MLST) or staphylococcal protein A (spa) typing. These methods determine meticulously selected markers suitable for genotyping, but only approximately $0.1 \%$ of the whole nucleotide sequence of the bacterium. The results of NGS/WGS (next generation sequencing/ whole genome shotgun) can cover more than $99 \%$ of the genetic information of the microorganism. 
Whole-genome multilocus sequence typing (wgMLST) is a genotyping method in which several thousand-virtually all known—species-specific alleles are determined from the whole genome of the microorganism. In the case of Staphylococcus aureus, the pan-genome MLST scheme in the BioNumerics v7.6 software used in this study contains a total number of 3897 wgMLST alleles [17]. A total of 1861 comprises the core genome, an additional 2036 accessory loci, and the traditional seven MLST (multilocus sequence typing) sequences add up to complete the pan genome scheme of 3904 loci. Each wgMLST allele that is called within the genome is given a single numerical value according to its nucleotide sequence when compared with the database. If the sequence is not identical with any of the previous results, a new number is assigned. The identical number means identical nucleotide sequences on the locus, the difference of any extent is compressed into a different number for that locus. The pattern of allele numbers can be used to create clusters with a numerical representation of similarity between samples. One method is the creation of a minimum-spanning tree network. A planar graph is created from the wgMLST data. Points represent the strains, and the edges represent the genetic distance-in our case, the number of different loci, 0-3904-between each pair of isolates. The minimum-spanning tree is achieved by only keeping the shortest edge for as many points as possible. A point can have multiple connections, but only if this edge is the shortest connection for the other connected point.

In this study, we performed WGS on 50 Staphylococcus strains originating from Hungarian rabbit farms and compared the results with previous sequencing projects on the pathogen, one of our own, and 12 from other authors. This research aimed at revealing genome-wide genetical differences among strains representing the three virulence types ( $\mathrm{HV}$, aHV and $\mathrm{LV}$, respectively) and to map the possible genetic relations between them. In the case of strains, which were identified only as Staphylococcus sp. before, the aim was to identify them more precisely.

\section{Materials and Methods}

\subsection{Bacterial Strains}

The bacterial strains used for the sequencing were isolated from deceased animals turned in by the farmers for necropsy and subsequent diagnostic investigations. No live animals were involved in this study. We reported the results of a large-scale survey in 2016, where we analysed the virulence type of 374 isolates [6]. We selected $15 \mathrm{HV}, 15 \mathrm{aHV}, 12 \mathrm{LV}$ and 8 Staphylococcus sp., a total of 50 isolates from our archive for WGS. The 16th HV isolate was kindly provided by Katleen Hermans (Ghent University, Ghent, Belgium) as a PCR positive control. This HV strain labelled Sp17 (Spain 17) shows all the properties determined typical of this genotype in previous studies: mixed biotype CV-C (crystal violet type C) [11], sensitive to phages of phage group II (3A, 3C, and 71), shows the multiplex PCR pattern specific for highly virulent $S$. aureus strains [4], and has pulsed-field gel electrophoresis type N2, and Staphylococcus aureus protein A (spa) type t645 [5]. The draft genome sequence of SP17 was published by our research group previously as National Center for Biotechnology Information (NCBI) GenBank LBCS00000000 [18]. Digital archives provided another 12 rabbit-related Staphylococcus aureus genome sequences [8,9]. Detailed information about the Staphylococcus aureus strains used in this study is presented in Table 1. 
Table 1. Epidemiological and genotypic details of the Staphylococcus aureus isolates of rabbit origin investigated with whole-genome sequencing. Geographic place of origin is provided, where only the country is available, it is in all capital letters. The virulence type is only provided for the strains collected by the authors. Vir.: virulence type determined by multiplex PCR. Year: year of isolation of the strain.

\begin{tabular}{|c|c|c|c|c|c|c|c|c|c|c|c|c|c|c|}
\hline & Accession & Year & Vir. & Origin & spa Type & spa Repeat Succession & pubST & $\operatorname{arcC}$ & aroE & glpF & gmk & pta & tpi & yqiL \\
\hline 1 & ERS4418242 & 2009 & $\mathrm{LV}$ & Dabas & Unknown & 07-23-21-17-34-34-34-34-34-34-34 & 6063 & 12 & 1 & 611 & 15 & 11 & 1 & 337 \\
\hline 2 & ERS4418234 & 2010 & LV & Vaskút & t1190 & 07-23-21-17-34-34-34-34-33-34 & 2855 & 12 & 1 & 1 & 15 & 11 & 1 & 337 \\
\hline 3 & ERS4418241 & 2009 & $\mathrm{LV}$ & Hetényegyháza & Unknown & 07-23-21-17-34-34-34-34-34-34-34-33-34 & 6083 & 12 & 1 & 1 & 480 & 11 & 1 & 337 \\
\hline 4 & ERS4418236 & 2010 & LV & Bükkösd & $\mathrm{t} 127$ & 07-23-21-16-34-33-13 & 1 & 1 & 1 & 1 & 1 & 1 & 1 & 1 \\
\hline 5 & ERS4418237 & 2010 & LV & Bükkösd & $\mathrm{t} 127$ & 07-23-21-16-34-33-13 & 1 & 1 & 1 & 1 & 1 & 1 & 1 & 1 \\
\hline 6 & ERS4418240 & 2009 & LV & Mezőtúr & $\mathrm{t} 4022$ & $07-23-21-17-34-33-34$ & 2855 & 12 & 1 & 1 & 15 & 11 & 1 & 337 \\
\hline 7 & ERS4418235 & 2014 & $\mathrm{LV}$ & Losonctamási & t1190 & $07-23-21-17-34-34-34-34-33-34$ & 2855 & 12 & 1 & 1 & 15 & 11 & 1 & 337 \\
\hline 8 & ERS4418230 & 2009 & $\mathrm{LV}$ & Kartal & t11218 & $07-23-21-17-34-34-34-34-34-34-33-34$ & 2855 & 12 & 1 & 1 & 15 & 11 & 1 & 337 \\
\hline 9 & ERS4418238 & 2011 & $\mathrm{LV}$ & Fülöpháza & $\mathrm{t} 2802$ & 07-23-21-17-34-34-34-33-34 & 96 & 12 & 1 & 1 & 15 & 11 & 1 & 40 \\
\hline 10 & ERS4418233 & 2012 & $\mathrm{LV}$ & Püspökhatvan & $\mathrm{t} 11218$ & $07-23-21-17-34-34-34-34-34-34-33-34$ & 6064 & 12 & 1 & 68 & 15 & 11 & 1 & 337 \\
\hline 11 & ERS4418231 & 2011 & $\mathrm{LV}$ & Galgamácsa & t11218 & $07-23-21-17-34-34-34-34-34-34-33-34$ & 2855 & 12 & 1 & 1 & 15 & 11 & 1 & 337 \\
\hline 12 & ERS4418232 & 2011 & $\mathrm{LV}$ & Galgamácsa & t11218 & $07-23-21-17-34-34-34-34-34-34-33-34$ & 2855 & 12 & 1 & 1 & 15 & 11 & 1 & 337 \\
\hline 13 & ERS4418208 & 2013 & $\mathrm{aHV}$ & Kardoskút & $\mathrm{t} 4770$ & $04-12-21-17-34-24-34-22-25$ & 5993 & 26 & 3 & 57 & 478 & 734 & 4 & 3 \\
\hline 14 & ERS4418206 & 2012 & $\mathrm{aHV}$ & Magfa & $\mathrm{t} 4770$ & $04-12-21-17-34-24-34-22-25$ & 5993 & 26 & 3 & 57 & 478 & 734 & 4 & 3 \\
\hline 15 & ERS4418201 & 2012 & $\mathrm{aHV}$ & Vitka & $\mathrm{t} 4770$ & $04-12-21-17-34-24-34-22-25$ & 5993 & 26 & 3 & 57 & 478 & 734 & 4 & 3 \\
\hline 16 & ERS4418211 & 2012 & $\mathrm{aHV}$ & Györköny & $\mathrm{t} 4770$ & $04-12-21-17-34-24-34-22-25$ & 5993 & 26 & 3 & 57 & 478 & 734 & 4 & 3 \\
\hline 17 & ERS4418200 & 2009 & $\mathrm{aHV}$ & Hetényegyháza & $\mathrm{t} 4770$ & $04-12-21-17-34-24-34-22-25$ & 5993 & 26 & 3 & 57 & 478 & 734 & 4 & 3 \\
\hline 18 & ERS4418197 & 2013 & $\mathrm{aHV}$ & Ócsa & t2407 & $11-12-12-12-21-17-34-24-34-22-25$ & 5993 & 26 & 3 & 57 & 478 & 734 & 4 & 3 \\
\hline 19 & ERS4418198 & 2013 & $\mathrm{aHV}$ & Ócsa & $\mathrm{t} 2407$ & $11-12-12-12-21-17-34-24-34-22-25$ & 5993 & 26 & 3 & 57 & 478 & 734 & 4 & 3 \\
\hline 20 & ERS4418209 & 2013 & $\mathrm{aHV}$ & Györköny & $\mathrm{t} 4770$ & $04-12-21-17-34-24-34-22-25$ & 5993 & 26 & 3 & 57 & 478 & 734 & 4 & 3 \\
\hline 21 & ERS4418207 & 2012 & $\mathrm{aHV}$ & Vitka & $\mathrm{t} 4770$ & $04-12-21-17-34-24-34-22-25$ & 5993 & 26 & 3 & 57 & 478 & 734 & 4 & 3 \\
\hline 22 & ERS4418202 & 2011 & $\mathrm{aHV}$ & Kartal & $\mathrm{t} 4770$ & $04-12-21-17-34-24-34-22-25$ & 5993 & 26 & 3 & 57 & 478 & 734 & 4 & 3 \\
\hline 23 & ERS4418210 & 2013 & $\mathrm{aHV}$ & Alsótold & $\mathrm{t} 4770$ & $04-12-21-17-34-24-34-22-25$ & 5993 & 26 & 3 & 57 & 478 & 734 & 4 & 3 \\
\hline 24 & ERS4418203 & 2011 & $\mathrm{aHV}$ & Hetényegyháza & $\mathrm{t} 4770$ & $04-12-21-17-34-24-34-22-25$ & 5993 & 26 & 3 & 57 & 478 & 734 & 4 & 3 \\
\hline 25 & ERS4418212 & 2012 & aHV & Püspökhatvan & $\mathrm{t} 711$ & $04-21-17-34-24-34-22-25$ & 5993 & 26 & 3 & 57 & 478 & 734 & 4 & 3 \\
\hline
\end{tabular}


Table 1. Cont.

\begin{tabular}{|c|c|c|c|c|c|c|c|c|c|c|c|c|c|c|}
\hline & Accession & Year & Vir. & Origin & spa Type & spa Repeat Succession & pubST & $\operatorname{arcC}$ & aroE & glpF & gmk & pta & tpi & yqiL \\
\hline 26 & ERS4418199 & 2013 & $\mathrm{aHV}$ & Ócsa & $\mathrm{t} 2407$ & $11-12-12-12-21-17-34-24-34-22-25$ & 5993 & 26 & 3 & 57 & 478 & 734 & 4 & 3 \\
\hline 27 & ERS4418205 & 2011 & $\mathrm{aHV}$ & Galgamácsa & $\mathrm{t} 4770$ & $04-12-21-17-34-24-34-22-25$ & 5993 & 26 & 3 & 57 & 478 & 734 & 4 & 3 \\
\hline 28 & ERS4418221 & 2013 & $\mathrm{HV}$ & Györköny & t645 & $14-44-13-12-17-23-18-17$ & 121 & 6 & 5 & 6 & 2 & 7 & 14 & 5 \\
\hline 29 & ERS4418222 & 2013 & $\mathrm{HV}$ & Györköny & t645 & $14-44-13-12-17-23-18-17$ & 121 & 6 & 5 & 6 & 2 & 7 & 14 & 5 \\
\hline 30 & ERS4418227 & 2013 & $\mathrm{HV}$ & Györköny & t645 & $14-44-13-12-17-23-18-17$ & 121 & 6 & 5 & 6 & 2 & 7 & 14 & 5 \\
\hline 31 & ERS4418228 & 2013 & $\mathrm{HV}$ & Györköny & t645 & $14-44-13-12-17-23-18-17$ & 121 & 6 & 5 & 6 & 2 & 7 & 14 & 5 \\
\hline 32 & ERS4418224 & 2014 & $\mathrm{HV}$ & Györköny & t645 & $14-44-13-12-17-23-18-17$ & 121 & 6 & 5 & 6 & 2 & 7 & 14 & 5 \\
\hline 33 & ERS4418225 & 2014 & $\mathrm{HV}$ & Györköny & t645 & $14-44-13-12-17-23-18-17$ & 121 & 6 & 5 & 6 & 2 & 7 & 14 & 5 \\
\hline 34 & ERS4418214 & 2009 & $\mathrm{HV}$ & Cegléd & t645 & $14-44-13-12-17-23-18-17$ & 121 & 6 & 5 & 6 & 2 & 7 & 14 & 5 \\
\hline 35 & ERS4418219 & 2012 & $\mathrm{HV}$ & Magfa & t645 & $14-44-13-12-17-23-18-17$ & 121 & 6 & 5 & 6 & 2 & 7 & 14 & 5 \\
\hline 36 & ERS4418216 & 2011 & $\mathrm{HV}$ & Harta & t645 & $14-44-13-12-17-23-18-17$ & 121 & 6 & 5 & 6 & 2 & 7 & 14 & 5 \\
\hline 37 & ERS4418217 & 2011 & $\mathrm{HV}$ & Harta & t645 & $14-44-13-12-17-23-18-17$ & 121 & 6 & 5 & 6 & 2 & 7 & 14 & 5 \\
\hline 38 & ERS4418218 & 2011 & $\mathrm{HV}$ & Harta & t645 & $14-44-13-12-17-23-18-17$ & 121 & 6 & 5 & 6 & 2 & 7 & 14 & 5 \\
\hline 39 & ERS4418226 & 2011 & $\mathrm{HV}$ & Harta & t645 & $14-44-13-12-17-23-18-17$ & 121 & 6 & 5 & 6 & 2 & 7 & 14 & 5 \\
\hline 40 & ERS4418220 & 2012 & $\mathrm{HV}$ & Györköny & t645 & $14-44-13-12-17-23-18-17$ & 121 & 6 & 5 & 6 & 2 & 7 & 14 & 5 \\
\hline 41 & ERS4418223 & 2013 & $\mathrm{HV}$ & Alsótold & t645 & $14-44-13-12-17-23-18-17$ & 121 & 6 & 5 & 6 & 2 & 7 & 14 & 5 \\
\hline 42 & ERS4418215 & 2010 & $\mathrm{HV}$ & Ócsa & t645 & $14-44-13-12-17-23-18-17$ & 121 & 6 & 5 & 6 & 2 & 7 & 14 & 5 \\
\hline 43 & LBCS00000000 & 2004 & $\mathrm{HV}$ & SP17-SPAIN & t645 & $14-44-13-12-17-23-18-17$ & 121 & 6 & 5 & 6 & 2 & 7 & 14 & 5 \\
\hline 44 & ERR387096 & 2013 & - & Manchester & $\mathrm{t} 021$ & $15-12-16-02-16-02-25-17-24$ & 30 & 2 & 2 & 2 & 2 & 6 & 3 & 2 \\
\hline 45 & ERR387097 & 2013 & - & Manchester & t021 & $15-12-16-02-16-02-25-17-24$ & 30 & 2 & 2 & 2 & 2 & 6 & 3 & 2 \\
\hline 46 & ERR387166 & 2013 & - & ENGLAND & t1614 & 08-16-34-24-34-34-17-17-17 & 3126 & 3 & 37 & 19 & 2 & 20 & 369 & 32 \\
\hline 47 & ERR387196 & 1999 & - & SCOTLAND & t645 & $14-44-13-12-17-23-18-17$ & 121 & 6 & 5 & 6 & 2 & 7 & 14 & 5 \\
\hline 48 & ERR387195 & 1998 & - & SCOTLAND & t13114 & $14-44-12-23-18-17-17$ & 3120 & 18 & 33 & 6 & 20 & 7 & 335 & 48 \\
\hline 49 & ERR425000 & 2007 & - & SPAIN 06 & t645 & $14-44-13-12-17-23-18-17$ & 121 & 6 & 5 & 6 & 2 & 7 & 14 & 5 \\
\hline 50 & ERR425012 & 2003 & - & SPAIN 03 & t645 & $14-44-13-12-17-23-18-17$ & 121 & 6 & 5 & 6 & 2 & 7 & 14 & 5 \\
\hline 51 & ERR425013 & 2010 & - & ITALY & t645 & $14-44-13-12-17-23-18-17$ & 121 & 6 & 5 & 6 & 2 & 7 & 14 & 5 \\
\hline 52 & ERR494744 & 2009 & - & Stirlingshire & t15410 & $15-44-12-12-17-23-18-17-17-17-17-23-24$ & 3092 & 18 & 473 & 6 & 20 & 7 & 50 & 48 \\
\hline 53 & ERR494745 & 2012 & - & Glasgow & t15409 & $15-12-16-02-22-12-16-02-17-24$ & 39 & 2 & 2 & 2 & 2 & 2 & 2 & 2 \\
\hline 54 & ERR494746 & 2012 & - & Glasgow & t1977 & $26-16-28$ & 2257 & 7 & 6 & 1 & 5 & 30 & 8 & 6 \\
\hline
\end{tabular}




\subsection{Isolation of Strains, DNA and Library Preparation}

The strains were isolated from the infected organs using standard methods [19]. The isolates that were identified as Staphylococcus sp. had the following characteristics: Gram-positive, catalase-positive, oxidase-negative cocci with clustered aggregations, forming medium-sized, haemolysing, yellow or greyish pigment-producing colonies. Staphylococcus aureus species and virulence type were determined with multiplex PCR [4]. Strains were archived at $-80{ }^{\circ} \mathrm{C}$ using standard methods [20].

Genomic DNA from the Staphylococcus cultures was isolated using the NucleoSpin Microbial DNA Kit (Macherey-Nagel, Dueren, Germany) according to the manufacturer's instructions. The quality and quantity of the isolated DNA was assessed by measurements using a Qubit 4.0 fluorometer (Invitrogen, Waltham, MA, USA) and Tapestation 4150 systems (Agilent, Santa Clara, CA, USA).

The NGS libraries were prepared using the Nextera DNA Flex Library Prep Kit (Illumina, Eindhoven, The Netherlands) with Nextera DNA CD Indexes. The NGS libraries were sequenced on an Illumina MiSeq instrument using the MiSeq Reagent Kit v3 using paired-end 300 bp reads. Libraries were scaled to exhibit at least 100x. All raw sequencing data are available in the European Nucleotide Archive (ENA) under the project number PRJEB37661 and ERR4017499-ERR4017552 individual accession numbers.

\subsection{Traditional Genotyping}

The traditional MLST for Staphylococcus aureus targets seven house-keeping genes. Regions within $\operatorname{arcC}$ (Carbamate kinase), aroE (Shikimate dehydrogenase), glpF (Glycerol kinase), gmk (Guanylate kinase), pta (Phosphate acetyltransferase), tpi (Triosephosphate isomerase), yqiL (Acetyle coenzyme A acetyltransferase) coding regions were amplified and determined with Sanger sequencing. The result was compared with the PubMLST database, and each locus was converted into a single numerical value to determine the MLST sequence type (ST) of the strains [21,22]. The multivariable region within the Staphylococcus protein A coding gene was amplified with standard primers [23]. Both the MLST and spa types were assigned using the Bionumerics v7.6 software.

\subsection{Bioinformatic Analysis}

The fastq files were imported directly from Illumina BaseSpace to the BioNumerics version 7.6 software's (Applied Maths NV, Belgium) cloud-based calculation engine. De novo sequence assemblies were made with the SPAdes de novo genome assembler (version 3.7.1) [24]. The raw reads (fastq files) and the de novo assembled genome of each isolate were submitted to the BioNumerics' S. aureus wgMLST scheme for assembly-free (AF) and assembly-based (AB) wgMLST allele calling. The quality of the sequence read sets, de novo assemblies, and assembly-free and assembly-based allele calls, were assessed using the quality statistics window in BioNumerics. The wgMLST scheme contained 3897 wgMLST loci and the 7 traditional MLST loci, the allele sequences were matched with a database, and each allele was translated into a single numerical value. Traditionally, 7-gene MLST typing and spa typing were also determined from de novo assembled genomes and were also confirmed by Sanger sequencing.

A whole-genome MLST-based minimum spanning tree graph (MST) was constructed to infer the genetic distance of the isolates. MST is a planar, edge-weighted undirected graph, which connects all the points together, without any cycles, and with the minimum possible total edge length. All the distances are calculated between points, and the shortest distance is kept for each point.

For the annotation of draft genomes, we used RASTtk [25] and similar genomes were identified using the Similar Genome Finder service of PATRIC 3.6.3 [26].

\section{Results}

The WGS produced data files with excellent quality. The quality score on all bases was 35-36, with an average of 35.57. The percentage of bases with 30 or higher quality score (Q30\%) was 89-94\%. 
Coverage was 103.0-246.0, with an average of 156.6. Reads were assembled to $25-84$ contigs (median 41), the median contig size in genomes was $120-610 \mathrm{kbp}$ (thousand base pair). The $2683-2862 \mathrm{kbp}$ draft genomes were constructed de novo with 103-246 average coverage. Draft genome sequences will be provided on reasonable request.

The analysis of the draft genome sequences confirmed that the identification of 43 Staphylococcus aureus strains was correct. All HV strains belonged to the ST121 type and a t645 spa (Staphylococcus protein A) type (Table 1), as well as one isolate from Scotland, and the strains from Spain and Italy, according to previous reports about HV Staphylococcus aureus strains [2,5,8]. All aHV strains showed a novel MLST allele combination (deposited by the authors as ST5993 in the pubMLST database) because of unique gmk gene (encoding guanylate kinase) and $p t a$ gene (encoding phosphate acetyltransferase) sequences. The aHV strains were mostly spa type $t 4770$ (73\%), and one was the very similar t711 type. All these strains originated from a group of farms where the breeding animals were provided by the same genetic centre. Three other aHV strains $(20 \%)$ had the t2407 spa type and these isolates were cultured from diseased rabbits from the same Ócsa production unit.

LV strains make up five different MLST patterns and seven different spa types. Six strains (50\%) had been identified as ST2855. Three ST types and two spa types were not identified before. The three novel MLST sequence types, ST6063, ST6064 and ST6083, had been deposited by the authors in the pubMLST database. Two strains belonged to the very first MLST group (ST1), originating from the same isolated small-scale rural unit (Bükkösd). All the LV strains had identical aroE and tpi sequences, and $\operatorname{arcC}$ and $p t a$ were also homologous in all but the ST1 strains. Each of the three novel MLST sequence types (ST6063, ST6064 and ST6083) only differ by just one nucleotide from the major ST2855 type of the LV strains. The ST2855 strains comprise three different spa types: three t11218, two t1190 and one t4022. The ST6064 strain shared the t11218 spa type, and however the two other new ST types revealed novel spa types, but their repeats differed only by one repeat from the major spa type of t11218. The ST1 strains were t127 and the ST96 strain was t2802 spa sequence type (Table 1.).

The MST network constructed from the wgMLST analysis classified the Staphylococcus aureus strains from Hungary into four main clusters: $\mathrm{HV}, \mathrm{aHV}, \mathrm{LV}$ and the two ST1 isolates from Bükkösd formed a separate cluster (Figure 1). The length of the edge and the indicated number show how many loci are neighbouring strains apart, and the edge length uses logarithmic scaling of the distance. Except for the ST121 strains, the sequences derived from previous studies were very distant from the three virulent types of $\mathrm{HV}$, aHV and LV. The minimum spanning tree visualization shows that the strains from previous studies, as well as the groups of the three virulence types, have a marked distance of ca. 1730-1800 allelic difference (ca. $45 \%$ of all examined loci) from the centrally located Scotland-1998 strain. This isolate showed only 150 loci difference from the Stirlingshire isolate thus these two strains could be interpreted as one group. The two Manchester strains also formed a separate cluster (Figure 1).

The ST121 strains formed a distinguished group with inner distances ranging from 4-146 allelic differences. The average pairwise distance of 12 Hungarian HV ST121 isolates was 11.5 allelic difference, which is significantly less than the proposed clonality threshold of 24 alleles for Staphylococcus aureus [27]. Three Hungarian HV isolates (34-Cegléd, 41-Alsótold and 42-Ócsa) were also part of the ST121 clade but showed a distance of $>100$ allelic difference (Figure 1).

The aHV strains form a similarly distinct cluster from the other groups. The wgMLST revealed polymorphism on an average of 21.75 loci, which represents $0.55 \%$ of the complete set of all examined genetic features. Within one spa type (t4770), the allelic difference was 12-29 (0.3-0.7\%) alleles, while the t711 strain had $37(0.95 \%)$ and the $\mathrm{t} 2407$ had $64(1.6 \%)$ different alleles from the closest $\mathrm{t} 4770$ strain. LV strains grouped into two main clusters, 10 out of 12 strains clustered as a distinct group, but the distance of the individual isolates was greater than in the case of the HV and aHV isolates, which is according to the variance in the MLST and spa types within this virulence type. The two ST1 strains (4-Bükkösd and 5-Bükkösd) completely separated from all the strains and the clonal groups on the MST. Apart from the isolates form Bükkösd, the 9-Fülöpháza strain appears as a bit of an outlier among the LV strains with a distance of 321 alleles from the closest LV strain. The remaining nine LV isolates 
possess an average pairwise difference of 52.4 alleles, which is significantly more than in the case of either the HV or the aHV strains.

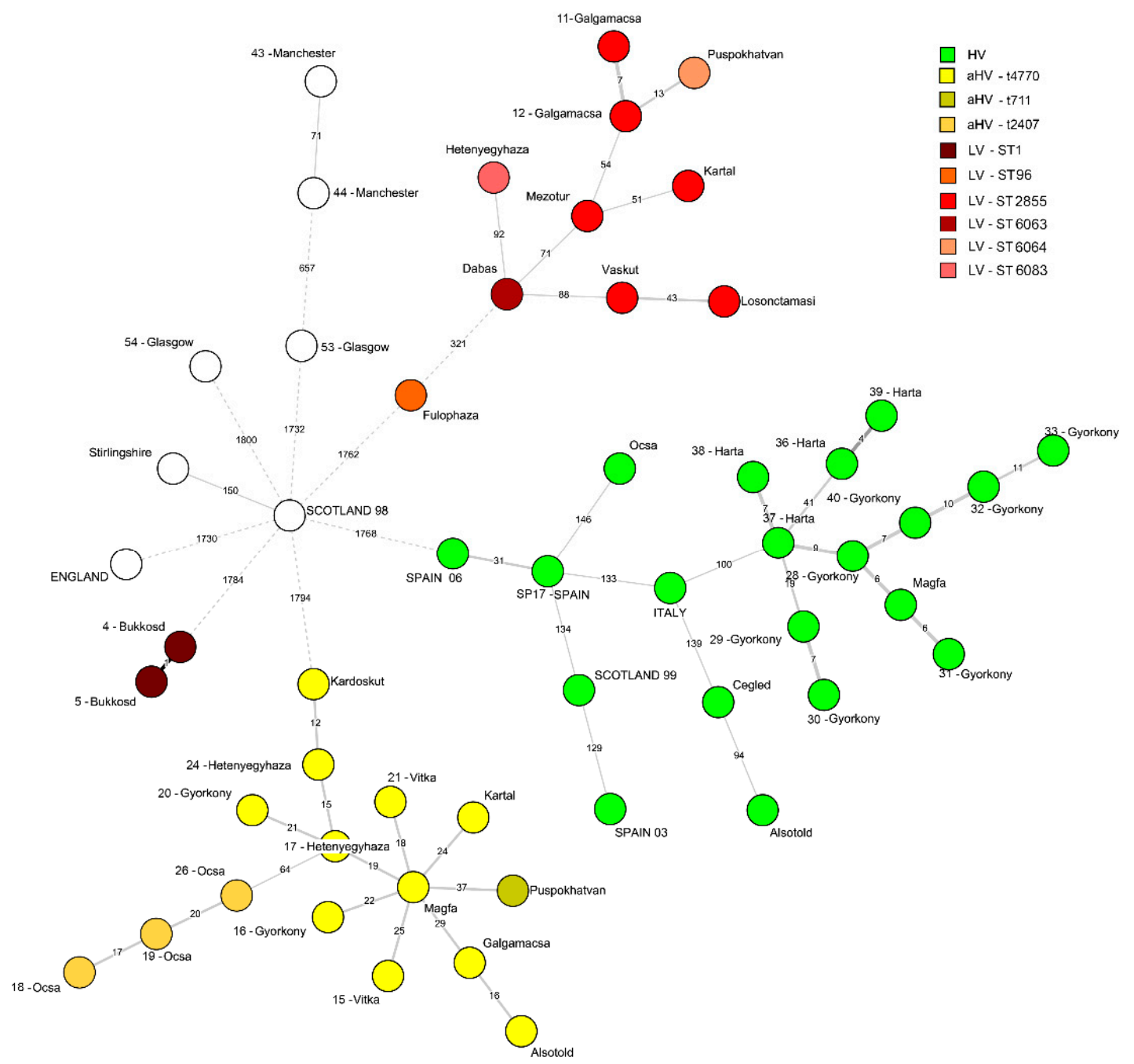

Figure 1. A minimum-spanning tree based on the whole-genome multilocus sequence typing profiles of 54 Staphylococcus aureus isolates of rabbit origin. The minimum spanning tree graph (MST) was created with the BioNumerics v7.6 advanced cluster analysis tool, where branch labels represent the allelic distances shown on a logarithmic scale. The corresponding multilocus sequence typing (MLST) and spa types within a virulence cluster are indicated by different shades of colours. The strains are identified with the city or country (all capital letters) of origin. Isolates are numbered as indicated in Table 1 . In case there were multiple isolates from one country, the year or year/number is also provided.

The genomes published by Holmes et al. [9] (except the Scotland 1999, ST121/t645 strain) were very different from the groups described above. The two strains from Manchester were closely related, as they both shared ST30/t021 types as described before. The Stirlingshire ST3092/t15410 and the Scotland 1998 ST3120/t13114 strains were different only on 150 loci (3.8\%). The two strains from Glasgow and the ST3126/t1614 from England were different on 657-1800 (16.8-46.1\%) loci, so they appear as individual clusters on Figure 1.

The strains classified as Staphylococcus sp. were isolated from specific lesions of rabbits with standard methods, but no further examinations were conducted on them until this study. These samples were not included in the MST network, as wgMLST scheme is Staphylococcus aureus species-specific and would have failed to identify the wgMLST loci. The PATRIC Similar Genome Finder service resulted in hits of Staphylococcus saprophyticus, xylosus and cohnii. 


\section{Discussion}

Staphylococcosis is one of the most important infectious diseases challenging rabbit farmers and veterinarians in modern commercial rabbit meat production. Virulent genotypes can cause epidemics that call for radical solutions, so genotyping the pathogen is a crucial element of diagnostics. Our earlier study showed that a previously rarely isolated, atypical variant is the most common type among the isolates collected from Hungarian rabbit farms [6]. The multiplex PCR method used to differentiate the highly virulent strains [4] was only capable of indicating that two HV specific sequences were unable to be amplified from the aHV strains. The analysis of the whole genome of the Staphylococcus aureus isolates showed that HV, aHV and the majority of LV strains form separate genetic clusters with a high similarity of strains within each cluster, indicated by the allelic differences that support the clonal origin of the genotypes described 10-15 years ago.

The discriminatory power of the wgMLST method is outstanding: it examines 3904 loci of the pathogen. Some strains in this survey showed genetic polymorphism on more than 3500 loci compared to the centrally located strain with the largest distance from all clusters, and even within the same MLST ST group, hundreds of loci showed polymorphism, which can facilitate a very fine differentiation of isolates. The multiplex PCR method published in 2007 is reinforced by the results, since it can clearly differentiate high and low virulent isolates [4].

All ST121 HV strains formed a closely related cluster, and the Hungarian strains were very closely related to the strains originating from commercial producing units in Italy and Spain, and the strain isolated from a companion rabbit in 1999, in Scotland. Staphylococcus aureus ST121 is a globally disseminated hypervirulent clone, important in human medicine on every populated continent [28]. The clonal origin of rabbit HV strains was confirmed decades ago [5] and the significant pathogenicity of this genotype was experimentally proven [29,30]. It is clearly demonstrated that the infections on Hungarian rabbit farms are part of an epidemic spreading worldwide. Most European rabbit meat producers choose from a few hybrid breeds of rabbits, so it is not surprising that similar virulent pathogens could appear in distant countries. Most of our HV isolates originated from farms that are integrated in the production line of a rabbit meat company. The integrator has been providing breeding animals for the farms and most of them rely exclusively on this source for animals. Interestingly, one Hungarian HV isolate (42-Ócsa) is placed outside the other Hungarian ST121 isolate cluster (Figure 1.). The production unit of its origin had no contact with the aforementioned integration, and thus was able to sustain an independent supply of breeding animals. This finding demonstrated that ST121 HV strains found multiple ways to contaminate commercial rabbit production in Hungary.

The aHV genotypes are almost as closely related to each other as the strains of the HV cluster. It is well known that in closed, industrial rabbit populations, different Staphylococcus variants can cause health problems [2,5,6]. This genotype was detected in 1994 on four Belgian farms, where the rabbits originated from the Czech Republic [10]. In this case, the solution for the epidemic was the complete eradication of the affected populations, and since then this genotype was not reported until a Hungarian survey in $2014[6,10]$. Our results clarified that these isolates, until now only identified by the amplification of a few selected genetic elements, constitute a group of closely related, most probably clonal specimens of Staphylococcus aureus.

The aHV group presented a novel MLST sequence type (ST 5993). Besides the unique gmk and pta sequences, other ST alleles were also very rare variants. At the time of the study, 5993 S. aureus MLST profiles are listed in the PubMLST database, and among them only four and three contain the same allelic variant of $\operatorname{arcC}$ and $g l p F$, respectively [21].

The whole genome analysis of the LV strains also provided interesting new discoveries. LV strains were considered to be less pathogenic variants based on biochemical properties related to humanor poultry-associated strains. The minor cluster confirms this idea, but the major cluster suggests that these strains might represent a third rabbit-associated clonal lineage. The similarity between the three main clusters is very low, around $22.8 \%$ of all loci have the same allele between the aHV and LV strains, and only $6.4 \%$ between the HV-aHV or HV-LV genotypes. The majority of the strains 
formed a cluster of very closely related strains, despite the fact that both MLST and spa sequence typing methods resulted in great diversity. The traditional seven-gene MLST and the single spa sequence would have distributed these $10 \mathrm{LV}$ strains into seven different clusters, while the analysis of 3897 loci revealed that within the main cluster, only an average of $1.3 \%$ of the genetic features was different. Based on the wgMLST, the clonal origin of this cluster also seems very probable. The origin of the outlier ST96/t2802 LV strain from Fülöpháza remains unclear. The difference in MLST and spa coding sequences is only a few nucleotides, but the wgMLST showing four times more different loci than the average between other members of the LV group reinforced the distinctiveness of this variant. Further sequencing projects on LV strains might reveal other specimens of this sequence type.

The second branch within the LV genotype only contained two strains, isolated in the same diagnostic case from two different animals. This rabbitry is a small-scale rural unit, where technological problems and poor hygiene could have contributed to the infection of the animals. ST1 is an important variant of this pathogen in both human and veterinary medicine. The clonal lineage has low host specificity, thus the presence of such strains may imply human-originated contamination or poses a risk for the zoonotic transmission of the bacteria.

Attili et al. examined the genotype of $96 \mathrm{~S}$. aureus strains, all isolated at the same farm, but from both animals and farm workers. All of the strains were classified as LV, but similarly diverse, five different spa types were detected among these LV strains ( $\mathrm{t} 094, \mathrm{t} 491, \mathrm{t} 605, \mathrm{t} 2036, \mathrm{t} 2802)$, none of which were similar to the spa type of the strains from Hungary [3].

The different virulence types can coexist within the same farm, but they have never been isolated simultaneously from the same animal in our studies [6]. The result of whole-genome sequencing confirmed that these variants are different clonal types indeed, the strains within a virulence group are similar to each other, and very different from other genotypes presenting at the same time in the same production unit. This reinforces our previous finding about the coexistence of different virulence types of Staphylococcus aureus on rabbit farms. Microbes of the skin and mucosal microbiota compete with each other for space and resources and the interference between the different variants of the same pathogenic species would be an interesting topic for future research. Some variants could be less virulent but more successful in colonisation, and such strains could be useful to decrease the spread of more aggressive variants.

The WGS sequencing of Staphylococcus strains, which did not contain the femA gene, thus classified as Staphylococcus sp. before, promised the possibility of identifying new pathogenic species related to rabbit staphylococcosis [6]. However, the results showed that these strains were closely related to bacteria, which are members of the dermal microbiota, but are also known for their possible etiological role in dermatitis [31]. All of these strains were isolated from contaminated skin lesions, and their presence might be secondary to physical trauma of the dermis, but also could be the origin of the disease. Animal experimental models $[29,30]$ could decide this question, however, neither the negligible prevalence, nor the clinical importance of this infection could justify such research. Despite the questionable clinical importance, the results and the sequence data can be useful for further studies.

\section{Conclusions}

Next generation sequencing is clearly the future way of diagnostics and epidemiology. WGS in this project was conducted at a lower expense per sample when compared with the eight PCR reactions and sequencing needed for conventional MLST and spa typing cost. The PCR and Sanger sequencing methods remain useful for diagnostics where NGS equipment is not available, but their limitations are clearly demonstrated with the LV strains in this study. These strains are classified as several different MLST and spa sequence types, while whole-genome multilocus sequence typing revealed that these strains are very closely related.

Our results confirmed that several different genotypes of Staphylococcus aureus can cause clinical problems in commercial rabbit farms at the same time. Diagnostic procedures should always determine the genotype of the pathogen, preferably multiple isolates within a case, since differences in virulence types 
could determine radically different treatment and prevention strategies. Quarantining and monitoring imported breeding animals from new sources are the only actions that could prevent the contamination of the whole population with perilous pathogenic agents.

Author Contributions: Conceptualization, Z.N., E.A., Á.D., G.B. and I.B.; methodology, Z.N. and R.S.; software, Z.N., Á.S. and R.S.; validation, R.S. and Á.S.; formal analysis, Z.N. and R.S.; investigation, Z.N. and E.A.; resources, Z.N. and E.A.; data curation, Z.N., S.B. and Á.S. writing-original draft preparation, Z.N. and R.S.; writing-review and editing, Z.N., E.A., Á.D., G.B., Á.S., S.B. and I.B.; visualization, Z.N. and R.S.; supervision, Z.N. and Á.S.; project administration, Z.N. and Á.S.; funding acquisition, Z.N. and Á.S. All authors have read and agreed to the published version of the manuscript.

Funding: This project was implemented with the support provided by the National Research, Development and Innovation Fund of Hungary, financed under the 2017-1.3.1-VKE-2017-00026 project number. The publication is supported by the European Union and co-financed by the European Social Fund (grant agreement no. EFOP-3.6.3-VEKOP-16-2017-00005, project title: "Strengthening the scientific replacement by supporting the academic workshops and programs of students, developing a mentoring process").

Acknowledgments: We are very grateful for Katleen Hermans for the SP17 strain, and all the help she provided. David Viana also provided very useful information for our analysis.

Conflicts of Interest: The authors declare no conflict of interest. The funders had no role in the design of the study; in the collection, analysis, or interpretation of data; in the writing of the manuscript, or in the decision to publish the results.

\section{References}

1. Corpa, J.M.; Hermans, K.; Haesebrouck, E. Main pathologies associated with Staphylococcus aureus infections in rabbits: A review. World Rabbit Sci. 2010, 17, 115-125. [CrossRef]

2. Hermans, K.; Devriese, L.A.; Haesebrouck, F. Rabbit staphylococcosis: Difficult solutions for serious problems. Vet. Microbiol. 2003, 91, 57-64. [CrossRef]

3. Attili, A.-R.; Nebbia, P.; Bellato, A.; Galosi, L.; Papeschi, C.; Rossi, G.; Linardi, M.; Fileni, E.; Cuteri, V.; Chiesa, F.; et al. The Effect of Age and Sampling Site on the Outcome of Staphylococcus aureus Infection in a Rabbit (Oryctolagus cuniculus) Farm in Italy. Animals 2020, 10, 774. [CrossRef]

4. Vancraeynest, D.; Haesebrouck, F.; Hermans, K. Multiplex PCR assay for the detection of high virulence rabbit Staphylococcus aureus strains. Vet. Microbiol. 2007, 121, 368-372. [CrossRef]

5. Vancraeynest, D.; Haesebrouck, F.; Deplano, A.; Denis, O.; Godard, C.; Wildemauwe, C.; Hermans, K. International Dissemination of a High Virulence Rabbit Staphylococcus aureus Clone. J. Vet. Med. Ser. B 2006, 53, 418-422. [CrossRef]

6. Német, Z.; Albert, E.; Nagy, K.; Csuka, E.; Dán, Á.; Szenci, O.; Hermans, K.; Balka, G.; Biksi, I. Virulence type and tissue tropism of Staphylococcus strains originating from Hungarian rabbit farms. Vet. Microbiol. 2016, 193, 1-6. [CrossRef]

7. Matuszewska, M.; Murray, G.G.R.; Harrison, E.M.; Holmes, M.A.; Weinert, L.A. The Evolutionary Genomics of Host Specificity in Staphylococcus aureus. Trends Microbiol. 2020. [CrossRef]

8. Viana, D.; Comos, M.; McAdam, P.R.; Ward, M.J.; Selva, L.; Guinane, C.M.; González-Muñoz, B.M.; Tristan, A.; Foster, S.J.; Fitzgerald, J.R.; et al. A single natural nucleotide mutation alters bacterial pathogen host tropism. Nat. Genet. 2015, 47, 361-366. [CrossRef] [PubMed]

9. Holmes, M.A.; Harrison, E.M.; Fisher, E.A.; Graham, E.M.; Parkhill, J.; Foster, G.; Paterson, G.K. Genomic Analysis of Companion Rabbit Staphylococcus aureus. PLoS ONE 2016, 11. [CrossRef]

10. Devriese, L.A.; Hendrickx, W.; Godard, C.; Okerman, L.; Haesebrouck, F. A New Pathogenic Staphylococcus aureus Type in Commercial Rabbits. J. Vet. Med. Ser. B 1996, 43, 313-315. [CrossRef]

11. Devriese, L.A. A simplified system for biotyping Staphylococcus aureus strains isolated from different animal species. J. Appl. Bacteriol. 1984, 56, 215-220. [CrossRef]

12. Vancraeynest, D.; Hermans, K.; Haesebrouck, F. Genotypic and phenotypic screening of high and low virulence Staphylococcus aureus isolates from rabbits for biofilm formation and MSCRAMMs. Vet. Microbiol. 2004, 103, 241-247. [CrossRef] [PubMed] 
13. Vancraeynest, D.; Hermans, K.; Haesebrouck, F. Prevalence of genes encoding exfoliative toxins, leucotoxins and superantigens among high and low virulence rabbit Staphylococcus aureus strains. Vet. Microbiol. 2006, 117, 211-218. [CrossRef] [PubMed]

14. Viana, D.; Selva, L.; Segura, P.; Penadés, J.R.; Corpa, J.M. Genotypic characterization of Staphylococcus aureus strains isolated from rabbit lesions. Vet. Microbiol. 2007, 121, 288-298. [CrossRef] [PubMed]

15. Viana, D.; Selva, L.; Penadés, M.; Corpa, J.M. Screening of virulence genes in Staphylococcus aureus isolates from rabbits. World Rabbit Sci. 2015, 23, 185-195. [CrossRef]

16. Satta, G.; Ling, C.L.; Cunningham, E.S.; McHugh, T.D.; Hopkins, S. Utility and limitations of Spa-typing in understanding the epidemiology of staphylococcus aureus bacteraemia isolates in a single University Hospital. BMC Res. Notes 2013, 6, 398. [CrossRef]

17. Roisin, S.; Gaudin, C.; De Mendonça, R.; Bellon, J.; Van Vaerenbergh, K.; De Bruyne, K.; Byl, B.; Pouseele, H.; Denis, O.; Supply, P. Pan-genome multilocu s sequence typing and outbreak-specific reference-based single nucleotide polymorphism analysis to resolve two concurrent Staphylococcus aureus outbreaks in neonatal services. Clin. Microbiol. Infect. 2016, 22, 520-526. [CrossRef]

18. Német, Z.; Albert, E.; Nagy, T.; Olasz, F.; Barta, E.; Kiss, J.; Dán, Á.; Bányai, K.; Hermans, K.; Biksi, I. Draft Genome Sequence of a Highly Virulent Rabbit Staphylococcus aureus Strain. Genome Announc. 2015, 3, e00461-15. [CrossRef]

19. Quinn, P.J. (Ed.) Clinical Veterinary Microbiology; Mosby: Edinburgh, UK, 2000; ISBN 978-0-7234-1711-8.

20. Murray, P.R.; Baron, E.J.; American Society for Microbiology. Manual of Clinical Microbiology; ASM Press: Washington, DC, USA, 2003; ISBN 978-1-55581-255-3.

21. Jolley, K.A.; Bray, J.E.; Maiden, M.C.J. Open-access bacterial population genomics: BIGSdb software, the PubMLST.org website and their applications. Wellcome Open Res. 2018, 3, 124. [CrossRef]

22. Maiden, M.C.J. Multilocus Sequence Typing of Bacteria. Annu. Rev. Microbiol. 2006, 60, 561-588. [CrossRef]

23. Harmsen, D.; Claus, H.; Witte, W.; Rothgänger, J.; Claus, H.; Turnwald, D.; Vogel, U. Typing of methicillinresistant Staphylococcus aureus in a university hospital setting by using novel software for spa repeat determination and database management. J. Clin. Microbiol. 2003, 41, 5442-5448. [CrossRef] [PubMed]

24. Nurk, S.; Bankevich, A.; Antipov, D.; Gurevich, A.; Korobeynikov, A.; Lapidus, A.; Pribelsky, A.; Pyshkin, A.; Sirotkin, A.; Sirotkin, Y.; et al. Assembling Genomes and Mini-metagenomes from Highly Chimeric Reads. In Proceedings of the Research in Computational Molecular Biology; Deng, M., Jiang, R., Sun, F., Zhang, X., Eds.; Springer: Berlin/Heidelberg, Germany, 2013; pp. 158-170.

25. Brettin, T.; Davis, J.J.; Disz, T.; Edwards, R.A.; Gerdes, S.; Olsen, G.J.; Olson, R.; Overbeek, R.; Parrello, B.; Pusch, G.D.; et al. RASTtk: A modular and extensible implementation of the RAST algorithm for building custom annotation pipelines and annotating batches of genomes. Sci. Rep. 2015, 5, 8365. [CrossRef]

26. Wattam, A.R.; Davis, J.J.; Assaf, R.; Boisvert, S.; Brettin, T.; Bun, C.; Conrad, N.; Dietrich, E.M.; Disz, T.; Gabbard, J.L.; et al. Improvements to PATRIC, the all-bacterial Bioinformatics Database and Analysis Resource Center. Nucleic Acids Res. 2017, 45, D535-D542. [CrossRef] [PubMed]

27. Schürch, A.C.; Arredondo-Alonso, S.; Willems, R.J.L.; Goering, R.V. Whole genome sequencing options for bacterial strain typing and epidemiologic analysis based on single nucleotide polymorphism versus gene-by-gene-based approaches. Clin. Microbiol. Infect. 2018, 24, 350-354. [CrossRef]

28. Rao, Q.; Shang, W.; Hu, X.; Rao, X. Staphylococcus aureus ST121: A globally disseminated hypervirulent clone. J. Med. Microbiol. 2015, 64, 1462-1473. [CrossRef] [PubMed]

29. Hermans, K.; De Herdt, P.; Devriese, L.A.; Godard, C.; Haesebrouck, F. Colonisation of rabbits with Staphylococcus aureus after experimental infection with high and low virulence strains. Vet. Microbiol. 2000, 72, 277-284. [CrossRef]

30. Meulemans, G.; Haesebrouck, F.; Lipinska, U.; Duchateau, L.; Hermans, K. Efficacy of an autogenous vaccine against highly virulent Staphylococcus aureus infection in rabbits. World Rabbit Sci. 2011, 19. [CrossRef]

31. Argemi, X.; Hansmann, Y.; Prola, K.; Prévost, G. Coagulase-Negative Staphylococci Pathogenomics. Int. J. Mol. Sci. 2019, 20, 1215. [CrossRef]

(C) 2020 by the authors. Licensee MDPI, Basel, Switzerland. This article is an open access article distributed under the terms and conditions of the Creative Commons Attribution (CC BY) license (http://creativecommons.org/licenses/by/4.0/). 